\title{
POLARIZATION AND FREQUENCY-POLARIZATION DEPENDENCES OF THREE-PHOTON INTERBAND LINEAR-CIRCULAR DICHROISM IN SEMICONDUCTORS OF CUBIC SYMMETRY
}

\author{
Voxob Rustamovich Rasulov ${ }^{1}$, Rustam Yavkachovich Rasulov ${ }^{2}$, \\ Mavzurjon Xursandboyevich Qo'chqorov ${ }^{3}$, \\ Nurillo Ubaydullo o'g'li Kodirov ${ }^{4}$ \\ ${ }^{1}$ Docent,Department of Physics, Fergana State University,Uzbekistan. \\ ${ }^{2}$ Professor,Department of Physics, Fergana State University, Uzbekistan. \\ ${ }^{3}$ PhD Research Scholar,Department of Physics, Kokand state pedagogical institute, Uzbekistan \\ ${ }^{4}$ PhD student, Fergana state university, Uzbekistan.
}

Article DOI: https://doi.org/10.36713/epra9076

DOI No: 10.36713/epra9076

\begin{abstract}
ANNOTATION
The polarization and frequency-polarization dependences of the linear-circular dichroism and light absorption coefficients in semiconductors of cubic symmetry, caused by vertical three-photon optical transitions between the states of
\end{abstract} the spin-orbit splitting and conduction bands, are calculated.

KEY WORDS: three-photon optical transitions, spin-orbit splitting band, conduction band, linear-circular dichroism, light absorption, semiconductor.

\section{INTRODUCTION}

Currently, the main research in the field of multiphoton absorption of light is carried out in wide-gap semiconductors, since a number of their physicochemical properties have been studied in depth and in more detail. In this respect, multiphoton effects occurring in narrow-gap crystals have been little studied both in theoretical and experimental aspects. The main reason for this is that the theoretical study of a number of photon-kinetic phenomena in narrow-gap crystals requires the use of not only the Luttinger-Kohn approximation, but also the multiband Kane approach. In the latter case, theoretical calculations are performed using matrices of at least $6 \times 6$ or $8 \times 8$.

The first works on two-photon interband transitions in crystals were carried out in the early $1960 \mathrm{y}$., shortly after the appearance of lasers [1-3]. When calculating the matrix elements of two-photon transitions in crystals, perturbation theories were used in the field of an unpolarized electromagnetic wave [2, 3], where the two-band Kane model was used.

In [4-10], both theoretically and experimentally, linear-circular dichroism (LCD) of two- and threephoton absorption of light in crystals of cubic symmetry was investigated. However, the question of the polarization and frequency-polarization study of three-photon interband absorption of light in crystals of cubic symmetry, caused between the states of the spin-orbit splitting band and the conduction band in the Kane approximation, remained open. 


\section{POLARIZATION DEPENDENCE OF THREE-PHOTON LINEAR-CIRCULAR DICHROISM}

In our previous works, three-photon optical transitions originating from the state of the spin-orbit splitting subband into the conduction bands were analyzed and it was shown that they are 10 different transitions [11], which differ from each other by virtual states. As indicated in [11] (see formula (4)), the multiphoton interband light absorption coefficient has two terms, one of which describes the sum of partial light absorption coefficients, and the second contribution to the multiphoton light absorption coefficient due to the interference terms of the matrix elements of the above optical transitions in crystals of cubic symmetry.

First, we will analyze the polarization dependences of interband optical transitions arising from the state of the spin-orbit splitting subband $|\mathrm{SO}, \pm 1 / 2\rangle$ into $|c, \pm 1 / 2\rangle$ conduction bands (i.e., three-photon transitions of type $|\mathrm{SO}, \pm 1 / 2\rangle \Rightarrow|c, \pm 1 / 2\rangle$ ), where we take into account that for linearly polarized light:

$$
\left|e_{z}^{\prime}\right|^{2}=\cos ^{2} \alpha,\left|e_{ \pm}^{\prime}\right|^{2}=1-\left|e_{z}^{\prime}\right|^{2} \text {, for } \quad \text { circularly } \quad \text { polarized } \quad \text { light } \quad\left|e_{z}^{\prime}\right|^{2}=\frac{1}{2} \sin ^{2} \beta,
$$

$\left|e_{ \pm}^{\prime}\right|^{2}=1-\left|e_{z}^{\prime}\right|^{2} \mp P_{\text {circ }} \cos \beta$, where $\alpha(\beta)$ is the angle between the polarization vectors $\vec{e}$ (the wave vector of the photon $\vec{q}$ ) and the wave vector of current carriers $(\vec{k}), e_{x}^{\prime}, e_{y}^{\prime}, e_{z}^{\prime}$ are the projections of the polarization vector of light along the axis $x^{\prime}, y^{\prime}, z^{\prime}$, associated with the direction of the wave vector of electrons $\vec{k}\left(\vec{k} \| z^{\prime}\right), e_{ \pm}^{\prime}=e_{x}^{\prime} \pm i e_{y}^{\prime}, \vec{q}-$ is the wave vector of the photon, 99 is the degree of circular polarization of light, and the sign of " $\pm 1 / 2$ " is describes the spin states of current carriers.

Calculations carried out according to the golden rule of quantum mechanics [12] for InSb show that in three-photon transitions of the type $|\mathrm{SO}, \pm 1 / 2\rangle \Rightarrow|c, \pm 1 / 2\rangle$, the graph of the polarization dependence of the coefficient of three-photon linear-circular dichroism is described in Fig. 1, and if two virtual states lie in the subbands of the valence band, and Fig. 1, b, if both virtual states lie in the conduction band, and Fig. 1, c, if the initial virtual states lie in the conduction band, and the next ones, in the valence band (in the approximation quadratic in the wave vector in the effective carrier Hamiltonian, some of the optical transitions are forbidden , and the part is nonzero).

In quantitative calculations, the numerical values of the band parameters were taken from [13]. Note here that in the first case, the polarization dependence of three-photon linear-circular dichroism is almost independent of the frequency of light. This means that linear-circular dichroism does not appear in this case, i.e. interband three-photon absorption of light does not depend on the degree of polarization of the light.

For three-photon transitions of the type: if both virtual states lie in the valence subbands, then the graph of the polarization dependence of the coefficient of three-photon linear-circular dichroism is described in Fig. 1d. The polarization dependence of the linear-circular dichroism of three-photon absorption of light is determined in a similar way. The polarization dependence of the linear-circular dichroism of three-photon absorption of light is determined in a similar way.

Note here that the energy spectra of current carriers in all bands are taken as parabolic, and when the three branches of the band structure of the semiconductor are nonparabolic, then the quadratic dependence of the energy on the wave vector is approximately restored only near the edges of the Brillouin zones.

That. showed that the polarization dependence of the linear-circular dichroism of three-photon absorption of light on the type of optical transitions. Since the matrix elements of some optical transitions have in the numerator the difference in the energies of current carriers, which in a certain region of the frequency of light tends to zero, which leads to an anomalous increase in the spectral dependence of the three-photon absorption coefficient of light, but this phenomenon leads to a noticeable change in the spectral dependence of linear-circular dichroism, since the coefficient of linear-circular dichroism is defined as the ratio of the probabilities of optical transitions occurring upon absorption of light with linear and circular polarization. 

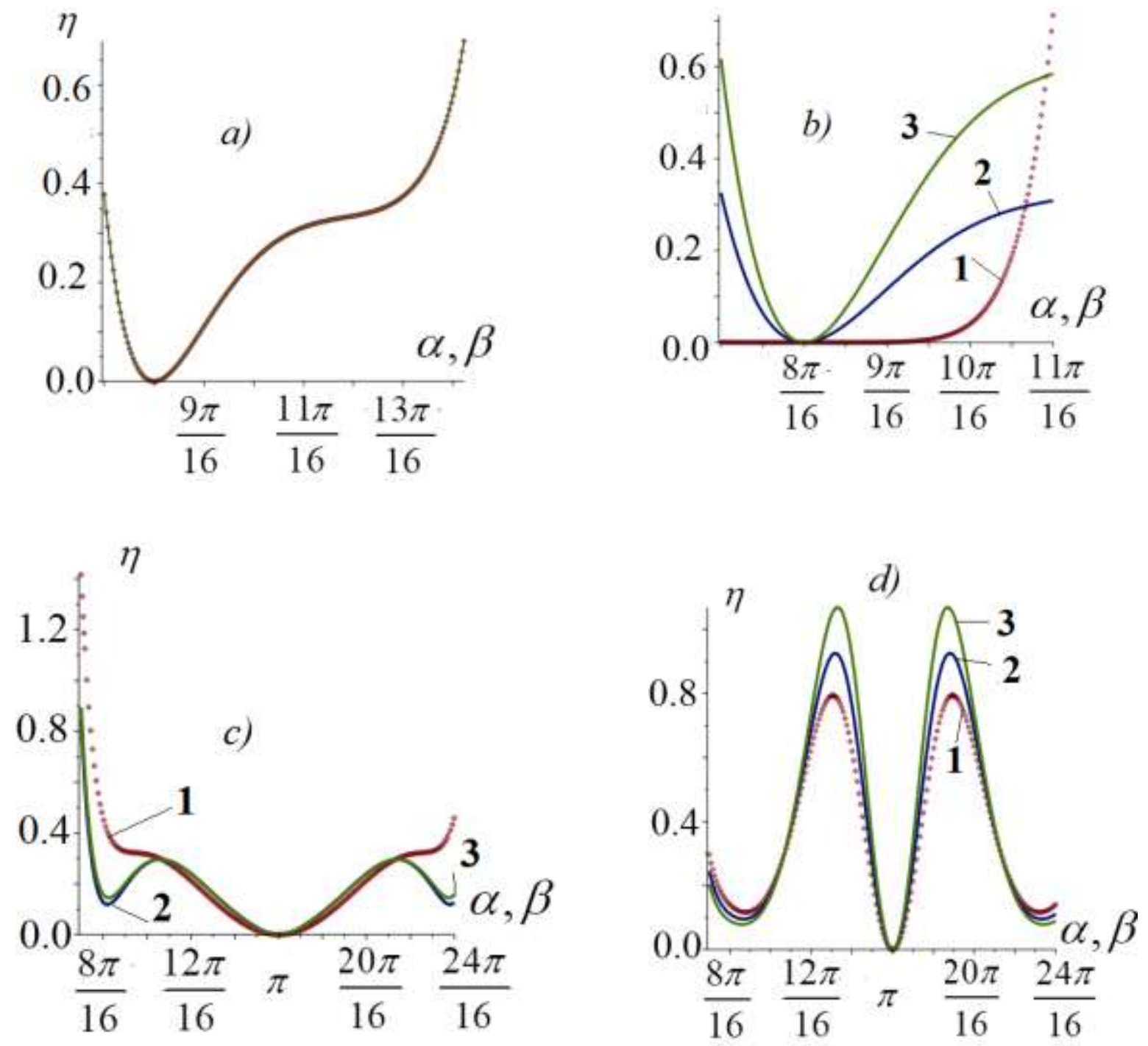

Fig. 1. Polarization dependences of three-photon linear-circular dichroism in InSb. Curves 1 correspond to the frequency of light $3 \hbar \omega=0,6 \mathrm{eV}$, curves 2 - $3 \hbar \omega=0,5 \mathrm{eV}$, and curves -

$$
3 \hbar \omega=0,44 e V .
$$

\section{FREQUENCY-POLARIZATION DEPENDENCES OF THE THREE-PHOTON LIGHT ABSORPTION COEFFICIENT}

Let us now analyze the frequency-spectral dependence of the light absorption coefficient for InSb, caused by three-photon transitions of the type. Calculations for the semiconductor InSb show that in threephoton transitions of the type, the graph of the polarization dependence of the coefficient of three-photon linearcircular dichroism is described in Fig. 2, when two virtual states lie in the subbands of the valence band, and Fig. 3, when both virtual states lie in the conduction band, and Fig. 4, if the initial virtual states lie in the conduction band, and the next ones lie in the valence band (in the approximation quadratic in the wave vector in the effective carrier Hamiltonian, some of the optical transitions are forbidden, and some nonzero). 


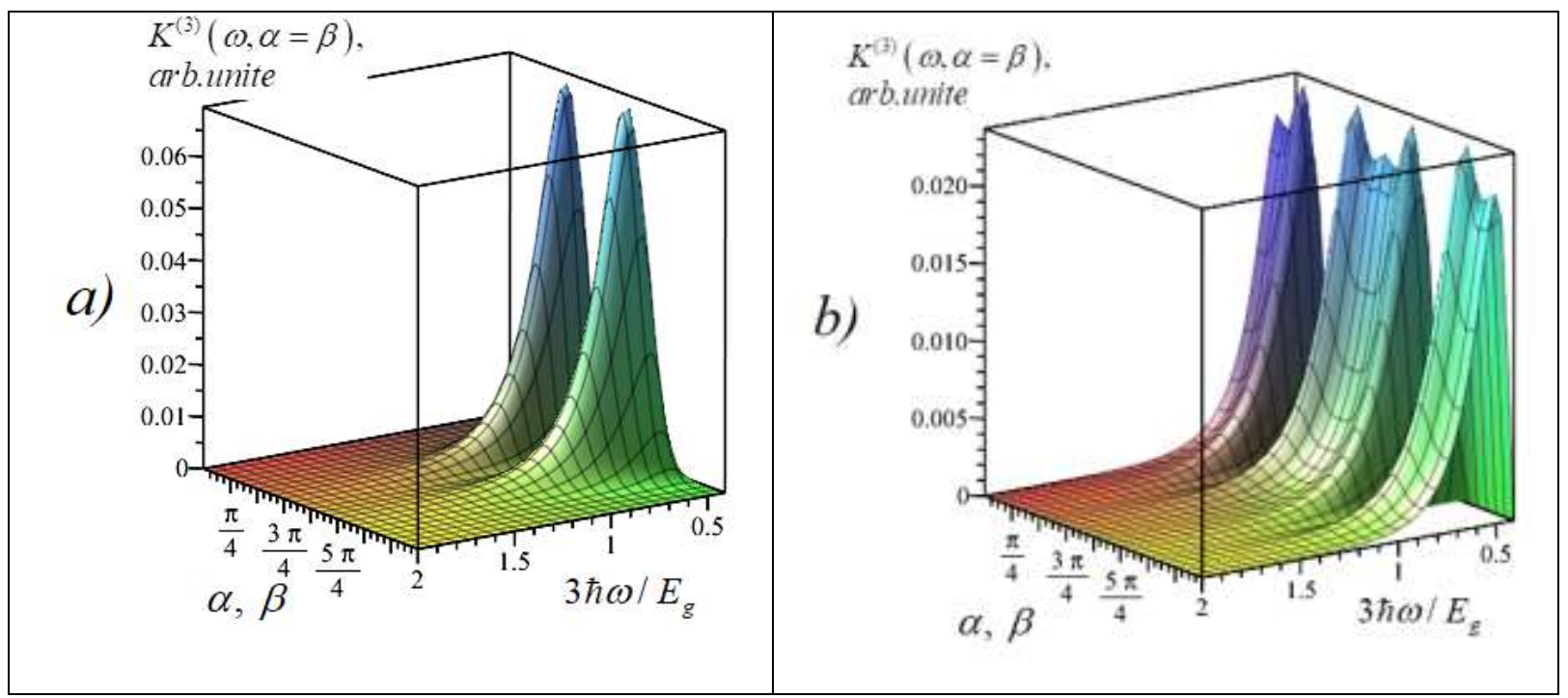

Fig. 2. Frequency-polarization dependences of the three-photon light absorption coefficient in GaAs. Curves a) correspond to circular polarization, curves b) to linear polarization.
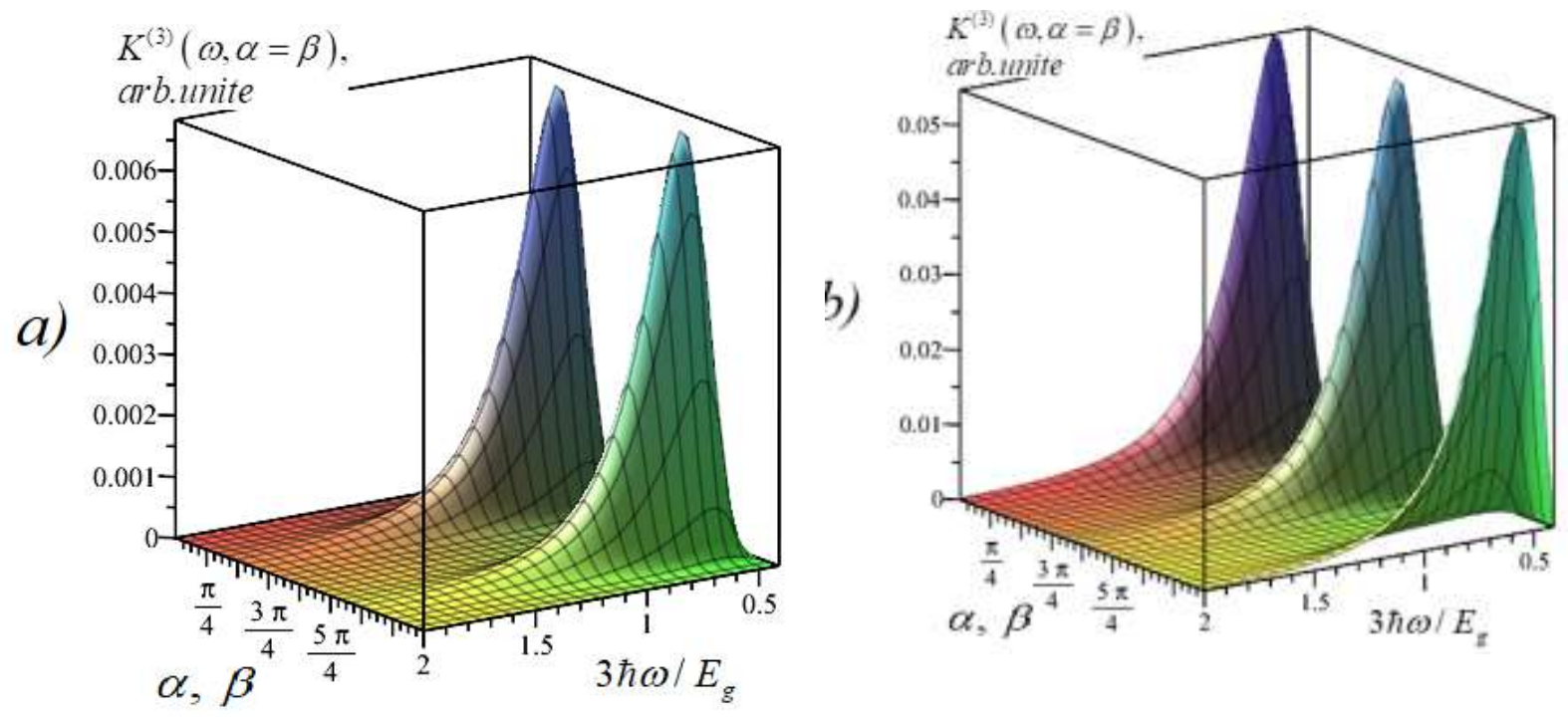

Fig. 3. Frequency-polarization dependences of the three-photon light absorption coefficient in GaAs. Curves a) correspond to circular polarization, curves b) to linear polarization. 

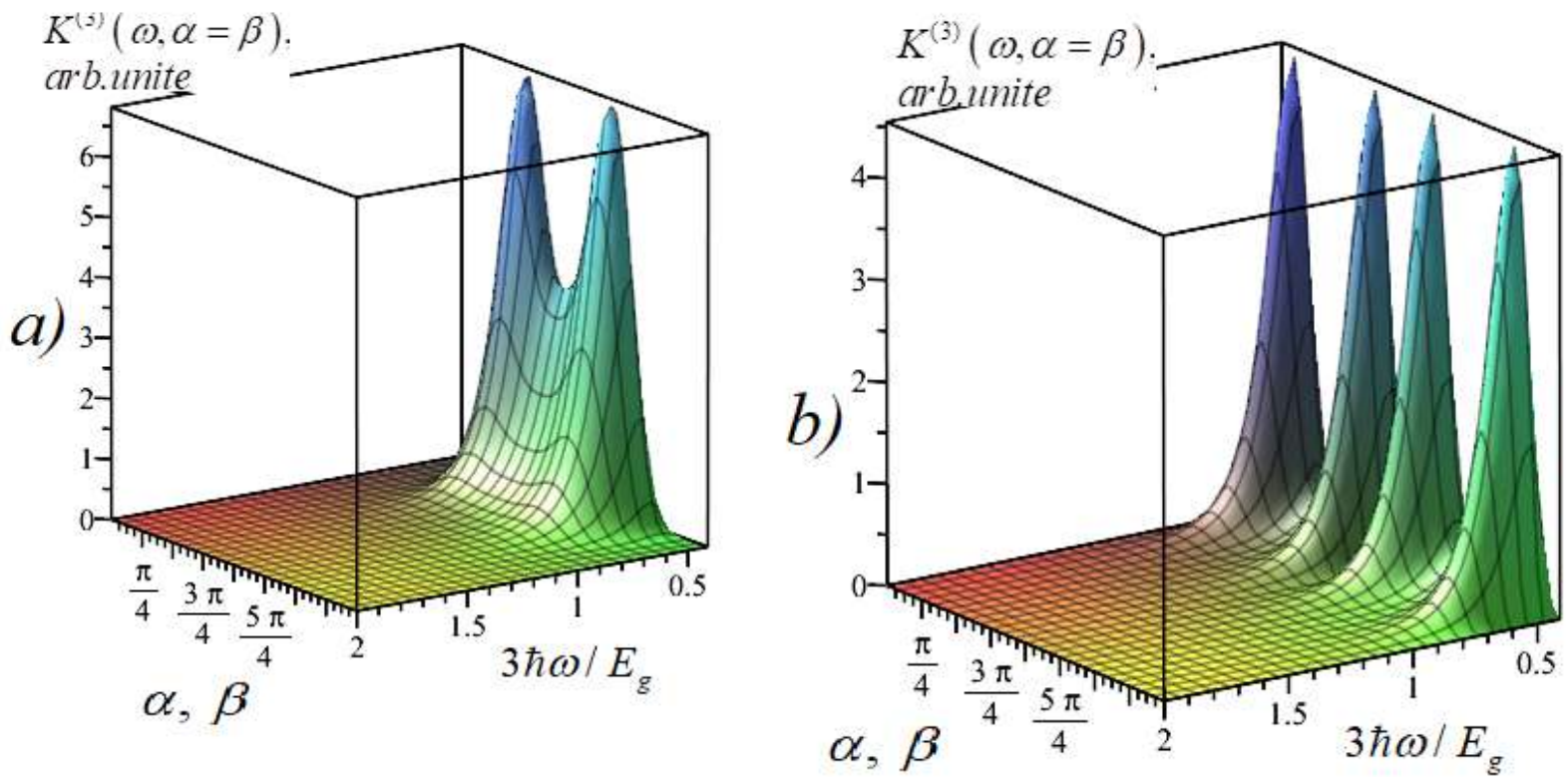

Fig. 4. Frequency-polarization dependences of the three-photon light absorption coefficient in GaAs. Curves a) correspond to circular polarization, curves b) to linear polarization.

It can be seen from Figs. 2-4 that the main contribution to the coefficient of three-photon absorption of light is made by optical transitions, in which the initial virtual states lie in the conduction band, and the next ones, in the valence band.

\section{CONCLUSION}

From the above results and Figures $1-4$, it can be seen that both the polarization and frequencypolarization dependences of the linear-circular dichroism and linear-circular dichroism coefficients have several extrema. This is due to the specificity of the Kane model used to study the band structure in narrow-gap crystals. In particular, in the Kane model, some off-diagonal matrix elements of the momentum operator do not depend on the wave vector of current carriers, which does not occur in the Luttinger-Kohn model.

\section{REFERENCES}

1. Miller A., A. Johnston, J. Dempsey, J. Smith, C. R. Pidgeon, and G. D. Holah, Two-photon absorption in InSb and Hg1-xCdxTe // - J. Phys. -1978. -No12.-Pp. 4839-4849.

2. Comparee C. R. Pidgeeon, B. S. Wherrett, A. M. Johnston, J. Dempsey, and A. Miller: Two-photon absorption in zinc-blende semiconductors // Phys. Rev. Lett.-1979. -Vol. 42.-Pp1785-1788 (1979), and references therein.

3. Braunstein R. and Ockman N. Optical double-photon absorption in CdS // - Phys. Rev. A. - 1964. -Vol. 34.-Pp. 499-507.

4. Rasulov R.Ya. Polarization optical VA photovoltaic effects in semiconductors with linear and nonlinear absorption of light. Dissertation for thesis. uch. doctor's degree phys.-math. sciences. St. Petersburg -1993. - 206 p. (in Russian)

5. Ivchenko E.L. Two-photon absorption and optical orientation of free carriers in cubic crystals // FTT. -1972.-T.14. Issue 12. -WITH. 3489-3485. (in Russian)

6. Beregulin E.V., Dvornikov D.P., Ivchenko E.L., Yaroshetskiy I.D. Polarization properties and linear-circular dichroism in nonlinear light absorption in $A_{2} B_{6}$ semiconductors // FTT. -1975. -T. 9. -No. 5.S. 876-886. (in Russian)

7. Arifzhanov S.B., Ivchenko E.L. Multiphoton absorption of light in crystals with a diamond and zinc blende structure // FTT. 1975. Vol. 17. \# 1.81-89 p. (in Russian)

8. Rasulov R.Ya. Linear circular dichroism in multiphoton interband absorption in semiconductors // FTT. - St.Petersburg, 1993. - T.35. - Issue 6. - Pp. 1674-1678. (in Russian)

9. Rasulov R.Ya. Linear-circular dichroism in multiphoton interband absorption in semiconductors // Solid State Physics. - St. Petersburg, 1993. - Vol. 35, - No. 6. - Pp. 1674-1677. (in Russian)

10. Rasulov V.R. Rasulov R.Ya., Eshboltaev I. Linearly and circular dichroism in a semiconductor with a complex valence band with allowance for four-photon absorption of light // Physics of the Solid State. - Springer, 2017. Vol.59. - No.3. - Pp. 463-468. 
11. Rasulov V. R., Rasulov R. Ya., Eshboltaev I. M., Qo'chqorov M. X. Interband Multiphoton Absorption of Light in Narrow-Gap Crystals // European Journal of Applied Physics. Vol.3, Issue5, 2021. DOI: http://dx.doi.org/10.24018/ejphysics.2021.3.5.ID

12. Landau L.D., Lifshits E.M. Quantum Mechanics (Nonrelativistic Theory) Vol. III. - M .. Fizmatlit, 2004 .- 800 p.(in Russian)

13. Vurgaftman I. and R. Meyer J., Ram-Mohan L. R. Band parameters for III - V compound semiconductors and their alloys // Journal of Applied Physics. 2001; 89 (11): 5815-5875. https://doi.org/10.1063/1.1368156. 